\title{
BIHARMONIC RIEMANNIAN SUBMERSIONS FROM 3-MANIFOLDS
}

\author{
ZE-PING WANG* AND YE-LIN OU**
}

\begin{abstract}
An important theorem about biharmonic submanifolds proved independently by Chen-Ishikawa [6] and Jiang [11 states that an isometric immersion of a surface into 3-dimensional Euclidean space is biharmonic if and only if it is harmonic (i.e, minimal). In a later paper [4, Cadeo-Monttaldo-Oniciuc shown that the theorem remains true if the target Euclidean space is replaced by a 3 -dimensional hyperbolic space form. In this paper, we prove the dual results for Riemannian submersions, i.e., a Riemannian submersion from a 3dimensional space form of non-positive curvature into a surface is biharmonic if and only if it is harmonic.
\end{abstract}

\section{INTRODUCTION AND THE MAIN RESULTS}

All manifolds, maps, tensor fields studied in this paper are assumed to be smooth unless there is an otherwise statement.

A biharmonic map is a map $\varphi:(M, g) \longrightarrow(N, h)$ between Riemannian manifolds that is a critical point of the bienergy

$$
E^{2}(\varphi, \Omega)=\frac{1}{2} \int_{\Omega}|\tau(\varphi)|^{2} \mathrm{~d} x
$$

for every compact subset $\Omega$ of $M$, where $\tau(\varphi)=$ Trace $_{g} \nabla \mathrm{d} \varphi$ is the tension field of $\varphi$ vanishing of which means the map is harmonic. By computing the first

Date: $02 / 23 / 2010$.

1991 Mathematics Subject Classification. 58E20, 53C43.

Key words and phrases. Biharmonic maps, Riemannian submersions, harmonic morphisms, 3-manifolds.

*Supported by Yunnan Wenshan University Research Project 09WSY03. The author is also grateful to the Department of Mathematics, Texas A \& M University-Commerce for the hospitality he received during a visit in Fall 2009 during which this work was done.

** Supported by Texas A \& M University-Commerce "Faculty Research Enhancement Project" (2009-10). 
variation of the functional (see [10]) one finds that $\varphi$ is biharmonic if and only if its bitension field vanishes identically, i.e.,

$$
\tau^{2}(\varphi):=\operatorname{Trace}_{g}\left(\nabla^{\varphi} \nabla^{\varphi}-\nabla_{\nabla^{M}}^{\varphi}\right) \tau(\varphi)-\operatorname{Trace}_{g} R^{N}(\mathrm{~d} \varphi, \tau(\varphi)) \mathrm{d} \varphi=0,
$$

where $R^{N}$ is the curvature operator of $(N, h)$ defined by

$$
R^{N}(X, Y) Z=\left[\nabla_{X}^{N}, \nabla_{Y}^{N}\right] Z-\nabla_{[X, Y]}^{N} Z .
$$

A submanifold is called a biharmonic submanifold if the isometric immersion that defines the submanifold is a biharmonic map. As biharmonic maps include harmonic maps as special cases biharmonic submanifols generalize the notion of minimal submanifolds (i.e., minimal isometric immersions). We use Proper biharmonic maps (respectively, submanifolds) to name those biharmonic maps (respectively, submanifolds) which are not harmonic.

A fundamental problem in the study of biharmonic maps is to classify all proper biharmonic maps between certain model spaces. An example of this is the following challenging conjecture which is still open.

Chen's Conjecture [5]: Any biharmonic isometric immersion $\left(M^{m}, g\right) \hookrightarrow \mathbb{R}^{n}$ into Euclidean space is harmonic.

Among several cases (see e.g., [7], [9]) that support the conjecture is the following theorem proved independently by Chen-Ishikawa [6] and Jiang [11].

Theorem. An isometric immersion $\left(M^{2}, g\right) \hookrightarrow \mathbb{R}^{3}$ into Euclidean space is biharmonic if and only if it is harmonic.

In a later paper [4], Cadeo-Monttaldo-Oniciuc shown that the theorem remains true if the target Euclidean space is replaced by a 3-dimensional hyperbolic space form.

In this paper, we prove the dual results for Riemannian submersions and give a complete classification of biharmonic Riemannian submersions from 3dimensional space form. Our main results can be stated as follows.

Theorem 3.3. Let $\pi:\left(M^{3}(c), g\right) \longrightarrow\left(N^{2}, h\right)$ be Riemannian submersion from a space form of constant sectional curvature $c$. Then, $\pi$ is biharmonic if and only if it is harmonic. 
Corollary 3.4. (1) If $\pi: \mathbb{R}^{3} \longrightarrow\left(N^{2}, h\right)$ is a biharmonic horizontally homothetic submersion from Euclidean space, then $\left(N^{2}, h\right)$ is flat and $\pi$ is a composition of an orthogonal projection $\mathbb{R}^{3} \longrightarrow \mathbb{R}^{2}$ followed by a covering map $\mathbb{R}^{2} \longrightarrow\left(N^{2}, h\right)$; (2) There exists no biharmonic Riemannian submersion $\pi: \mathbb{H}^{3} \longrightarrow\left(N^{2}, h\right)$ no matter what $\left(N^{2}, h\right)$ is.

Applying our results one can easily check the following

Example. The Riemannian submersion $\pi: \mathbb{R}^{3} \longrightarrow\left(N^{2}=\mathbb{R}^{3} / \mathbb{R}, h\right)$ from $\mathbb{R}^{3}$ onto the orbit space of a free 1-parameter isometric group action of $\mathbb{R}$ on $\mathbb{R}^{3}$ is not biharmonic, where the isometric group action is described by $(s,(z, t)) \longrightarrow$ $\left(e^{i s} z, t+s\right), s \in \mathbb{R},(z, t) \in \mathbb{C} \times \mathbb{R} \equiv \mathbb{C} \times \mathbb{R}$. In fact, if it were, then by our classification results, the Riemannian submersion would be harmonic and hence a harmonic morphism, and then a well-known theorem about harmonic morphisms (see, e.g., [1]) would imply that all fibers of $\pi$ would be geodesics, which is not the case.

\section{Biharmonic RiemanNian SUBMERSIONS From 3-MANifoldS}

In this section, we will describe biharmonicity of a Riemannian submersion from a generic 3-manifold by using the integrability data of a special orthonormal frame adapted to a Riemannian submersion. This is the main tool we use to prove our main theorem. We also construct a family of proper biharmonic Riemannian submersions from $\mathbb{R}^{3}$ provided with a warped product metric.

Let $\pi:\left(M^{3}, g\right) \longrightarrow\left(N^{2}, h\right)$ be a Riemannian submersion. A local orthonormal frame is said to be adapted to the Riemannian submersion $\pi$ if the vector fields in the frame that are tangent to the horizontal distribution are basic (i.e., they are $\pi$-related to a local orthonormal frame in the base space). Such a frame always exists (cf. e.g., [1]). Let $\left\{e_{1}, e_{2}, e_{3}\right\}$ be an orthonormal frame adapted to $\pi$ with $e_{3}$ being vertical. Then, it is well known (see [13]) that $\left[e_{1}, e_{3}\right]$ and $\left[e_{2}, e_{3}\right]$ are vertical and $\left[e_{1}, e_{2}\right]$ is $\pi$-related to $\left[\varepsilon_{1}, \varepsilon_{2}\right]$, where $\left\{\varepsilon_{1}, \varepsilon_{2}\right\}$ is an orthonormal frame in the base manifold. If we assume that

$$
\left[\varepsilon_{1}, \varepsilon_{2}\right]=F_{1} \varepsilon_{1}+F_{2} \varepsilon_{2}
$$

for $F_{1}, F_{2} \in C^{\infty}(N)$ and use the notations $f_{i}=F_{i} \circ \pi, i=1,2$. Then, we have

$$
\left\{\begin{array}{l}
{\left[e_{1}, e_{3}\right]=\kappa_{1} e_{3},} \\
{\left[e_{2}, e_{3}\right]=\kappa_{2} e_{3},} \\
{\left[e_{1}, e_{2}\right]=f_{1} e_{1}+f_{2} e_{2}-2 \sigma e_{3} .}
\end{array}\right.
$$


where $\kappa_{1}, \kappa_{2}$ and $\sigma \in C^{\infty}(M)$. We will call $f_{1}, f_{2}, \kappa_{1}, \kappa_{2}$ and $\sigma$ the integrability data of the adapted frame of the Riemannian submersion $\pi$.

Theorem 2.1. Let $\pi:\left(M^{3}, g\right) \longrightarrow\left(N^{2}, h\right)$ be a Riemannian submersion with the adapted frame $\left\{e_{1}, e_{2}, e_{3}\right\}$ and the integrability data $f_{1}, f_{2}, \kappa_{1}, \kappa_{2}$ and $\sigma$. Then, the Riemannian submersion $\pi$ is biharmonic if and only if

$$
\left\{\begin{array}{l}
-\Delta^{M} \kappa_{1}-f_{1} e_{1}\left(\kappa_{2}\right)-e_{1}\left(\kappa_{2} f_{1}\right)-f_{2} e_{2}\left(\kappa_{2}\right)-e_{2}\left(\kappa_{2} f_{2}\right) \\
+\kappa_{1} \kappa_{2} f_{1}+\kappa_{2}^{2} f_{2}+\kappa_{1}\left\{-K^{N}+f_{1}^{2}+f_{2}^{2}\right\}=0 \\
-\Delta^{M} \kappa_{2}+f_{1} e_{1}\left(\kappa_{1}\right)+e_{1}\left(\kappa_{1} f_{1}\right)+f_{2} e_{2}\left(\kappa_{1}\right)+e_{2}\left(\kappa_{1} f_{2}\right) \\
-\kappa_{1} \kappa_{2} f_{2}-\kappa_{1}^{2} f_{1}+\kappa_{2}\left\{-K^{N}+f_{1}^{2}+f_{2}^{2}\right\}=0
\end{array}\right.
$$

where $K^{N}=R_{1212}^{N} \circ \pi=-\left[e_{2}\left(f_{1}\right)-e_{1}\left(f_{2}\right)+f_{1}^{2}+f_{2}^{2}\right]$ is the Gauss curvature of Riemannian manifold $\left(N^{2}, h\right)$.

Proof. Let $\nabla$ denote the Levi-Civita connection of the Riemannian manifold $\left(M^{3}, g\right)$. A straightforward computation using (3) and Koszul formula gives

$$
\begin{aligned}
& \nabla_{e_{1}} e_{1}=-f_{1} e_{2}, \quad \nabla_{e_{1}} e_{2}=f_{1} e_{1}-\sigma e_{3}, \quad \nabla_{e_{1}} e_{3}=\sigma e_{2} \\
& \nabla_{e_{2}} e_{1}=-f_{2} e_{2}+\sigma e_{3}, \quad \nabla_{e_{2}} e_{2}=f_{2} e_{1}, \quad \nabla_{e_{2}} e_{3}=-\sigma e_{1} \\
& \nabla_{e_{3}} e_{1}=-\kappa_{1} e_{3}+\sigma e_{2}, \nabla_{e_{3}} e_{2}=-\sigma e_{1}-\kappa_{2} e_{3}, \nabla_{e_{3}} e_{3}=\kappa_{1} e_{1}+\kappa_{2} e_{2} .
\end{aligned}
$$

The tension of the Riemannian submersion $\pi$ is given by

$$
\tau(\pi)=\nabla_{e_{i}}^{\pi} d \pi\left(e_{i}\right)-d \pi\left(\nabla_{e_{i}}^{M} e_{i}\right)=-d \pi\left(\nabla_{e_{3}}^{M} e_{3}\right)=-\kappa_{1} \varepsilon_{1}-\kappa_{2} \varepsilon_{2}
$$

A straightforward computation using (5) yields

$$
\begin{aligned}
& \sum_{i=1}^{2} \nabla_{e_{i}}^{\pi} \nabla_{e_{i}}^{\pi} \tau(\pi)=\nabla_{e_{i}}^{\pi} \nabla_{e_{i}}^{\pi}\left(-\kappa_{1} \varepsilon_{1}-\kappa_{2} \varepsilon_{2}\right) \\
& =\left[-e_{1} e_{1}\left(\kappa_{1}\right)+\kappa_{1} f_{1}^{2}-f_{1} e_{1}\left(\kappa_{2}\right)-e_{1}\left(\kappa_{2} f_{1}\right)-e_{2} e_{2}\left(\kappa_{1}\right)\right. \\
& \left.+\kappa_{1} f_{2}^{2}-f_{2} e_{2}\left(\kappa_{2}\right)-e_{2}\left(\kappa_{2} f_{2}\right)\right] \varepsilon_{1} \\
& +\left[-e_{1} e_{1}\left(\kappa_{2}\right)+\kappa_{2} f_{1}^{2}+f_{1} e_{1}\left(\kappa_{1}\right)+e_{1}\left(\kappa_{1} f_{1}\right)-e_{2} e_{2}\left(\kappa_{2}\right)\right. \\
& \left.+\kappa_{2} f_{2}^{2}+f_{2} e_{2}\left(\kappa_{1}\right)+e_{2}\left(\kappa_{1} f_{2}\right)\right] \varepsilon_{2},
\end{aligned}
$$

$$
\nabla_{e_{3}}^{\pi} \nabla_{e_{3}}^{\pi} \tau(\pi)=-e_{3} e_{3}\left(\kappa_{1}\right) \varepsilon_{1}-e_{3} e_{3}\left(\kappa_{2}\right) \varepsilon_{2}
$$




$$
\begin{aligned}
& \sum_{i=1}^{3} \nabla_{\nabla_{e_{i}}^{M} e_{i}}^{\pi} \tau(\pi) \\
& =\nabla_{\nabla_{e_{1}}^{M} e_{1}}^{\pi}\left(-\kappa_{1} \varepsilon_{1}-\kappa_{2} \varepsilon_{2}\right)+\nabla_{\nabla_{e_{2}}^{M} e_{2}}^{\pi}\left(-\kappa_{1} \varepsilon_{1}-\kappa_{2} \varepsilon_{2}\right)+\nabla_{\nabla_{e_{3}}^{M} e_{3}}^{\pi}\left(-\kappa_{1} \varepsilon_{1}-\kappa_{2} \varepsilon_{2}\right) \\
(9) \quad & =\left[f_{1} e_{2}\left(\kappa_{1}\right)+\kappa_{2} f_{1} f_{2}-f_{2} e_{1}\left(\kappa_{1}\right)-\kappa_{2} f_{1} f_{2}-\kappa_{1} e_{1}\left(\kappa_{1}\right)\right. \\
& \left.-\kappa_{1} \kappa_{2} f_{1}-\kappa_{2} e_{2}\left(\kappa_{1}\right)-\kappa_{2}^{2} f_{2}\right] \varepsilon_{1} \\
& +\left[f_{1} e_{2}\left(\kappa_{2}\right)-\kappa_{1} f_{1} f_{2}-f_{2} e_{1}\left(\kappa_{2}\right)+\kappa_{1} f_{1} f_{2}+\kappa_{1}^{2} f_{1}-\kappa_{1} e_{1}\left(\kappa_{2}\right)\right. \\
& \left.+\kappa_{1} \kappa_{2} f_{2}-\kappa_{2} e_{2}\left(\kappa_{2}\right)\right] \varepsilon_{2},
\end{aligned}
$$

and

$$
\begin{aligned}
& \sum_{i=1}^{3} R^{N}\left(d \pi\left(e_{i}\right), \tau(\pi)\right) d \pi\left(e_{i}\right) \\
& =-\kappa_{1}\left\{e_{2}\left(f_{1}\right)-e_{1}\left(f_{2}\right)+f_{1}^{2}+f_{2}^{2}\right\} \varepsilon_{1} \\
& -\kappa_{2}\left\{e_{2}\left(f_{1}\right)-e_{1}\left(f_{2}\right)+f_{1}^{2}+f_{2}^{2}\right\} \varepsilon_{2} .
\end{aligned}
$$

Substituting equations (77)-(10) into the bitension field formula (10) we obtain

$$
\begin{aligned}
& \tau^{2}(\pi)=\sum_{i=1}^{3}\left\{\nabla_{e_{i}}^{\pi} \nabla_{e_{i}}^{\pi} \tau(\pi)-\nabla_{\nabla_{e_{i}}^{M} e_{i}}^{\pi} \tau(\pi)-R^{N}\left(d \pi\left(e_{i}\right), \tau(\pi)\right) d \pi\left(e_{i}\right)\right\} \\
& =\left[-\Delta^{M} \kappa_{1}-f_{1} e_{1}\left(\kappa_{2}\right)-e_{1}\left(\kappa_{2} f_{1}\right)-f_{2} e_{2}\left(\kappa_{2}\right)-e_{2}\left(\kappa_{2} f_{2}\right)\right. \\
& \left.+\kappa_{1} \kappa_{2} f_{1}+\kappa_{2}^{2} f_{2}+\kappa_{1}\left\{-K^{N}+f_{1}^{2}+f_{2}^{2}\right\}\right] \varepsilon_{1} \\
& +\left[-\Delta^{M} \kappa_{2}+f_{1} e_{1}\left(\kappa_{1}\right)+e_{1}\left(\kappa_{1} f_{1}\right)+f_{2} e_{2}\left(\kappa_{1}\right)+e_{2}\left(\kappa_{1} f_{2}\right)\right. \\
& \left.-\kappa_{1} \kappa_{2} f_{2}-\kappa_{1}^{2} f_{1}+\kappa_{2}\left\{-K^{N}+f_{1}^{2}+f_{2}^{2}\right\}\right] \varepsilon_{2},
\end{aligned}
$$

from which the theorem follows.

When the integrability data $\kappa_{2}=0$ we have the following corollary which will be used later in the paper.

Corollary 2.2. Let $\pi:\left(M^{3}, g\right) \longrightarrow\left(N^{2}, h\right)$ be a Riemannian submersion with an adapted frame $\left\{e_{1}, e_{2}, e_{3}\right\}$ and the integrability data $\left\{f_{1}, f_{2}, \kappa_{1}, \kappa_{2}, \sigma\right\}$ with $\kappa_{2}=0$. Then, the Riemannian submersion $\pi$ is biharmonic if and only if

$$
\left\{\begin{array}{l}
-\Delta^{M} \kappa_{1}+\kappa_{1}\left\{-K^{N}+f_{1}^{2}+f_{2}^{2}\right\}=0, \\
f_{1} e_{1}\left(\kappa_{1}\right)+e_{1}\left(\kappa_{1} f_{1}\right)+f_{2} e_{2}\left(\kappa_{1}\right)+e_{2}\left(\kappa_{1} f_{2}\right)-\kappa_{1}^{2} f_{1}=0,
\end{array}\right.
$$

Example 1. For $\varphi(x)=\frac{c_{1}\left(1+e^{c_{1} x}\right)}{1-e^{c_{1} x}}$, and $\phi(y)=\frac{b_{1}\left(1+e^{b_{1} y}\right)}{1-e^{b_{1} y}}$, and $\beta(x, y)=c e^{\int \varphi(x) d x+\int \phi(y) d y}$, the Riemannian submersion

$$
\begin{gathered}
\pi:\left(\mathbb{R}^{2} \times \mathbb{R}, d x^{2}+d y^{2}+\beta^{-2}(x, y) d z^{2}\right) \rightarrow\left(\mathbb{R}^{2}, d x^{2}+d y^{2}\right) \\
\phi(x, y, z)=(x, y)
\end{gathered}
$$

is a proper biharmonic map. In particular, when $\phi(y)=0$, the example recovers the family of proper biharmonic Riemannian submersion found in [12]. 
In fact, it is not difficult to check that the orthonormal frame $\left\{e_{1}=\frac{\partial}{\partial x}, e_{2}=\right.$ $\left.\frac{\partial}{\partial y}, \quad e_{3}=\beta \frac{\partial}{\partial z}\right\}$ on $\left(\mathbb{R}^{2} \times \mathbb{R}, d x^{2}+d y^{2}+\beta^{-2}(x, y) d z^{2}\right)$ is adapted to the Riemannian submersion $\pi$ with $d \pi\left(e_{i}\right)=\varepsilon_{i}, i=1,2$ and $e_{3}$ being vertical, where $\varepsilon_{1}=\frac{\partial}{\partial x}, \varepsilon_{2}=\frac{\partial}{\partial y}$, form an orthonormal frame on the base space $\left(\mathbb{R}^{2}, d x^{2}+d y^{2}\right)$. A straightforward computation gives the Lie brackets

$$
\left[e_{1}, e_{3}\right]=f e_{3},\left[e_{2}, e_{3}\right]=g e_{3}, \quad\left[e_{1}, e_{2}\right]=0,
$$

where $f=(\ln \beta)_{x}, g=(\ln \beta)_{y}$.

It follows that the integrability data of the Riemannian submersion $\pi$ are give by

$$
f_{1}=f_{2}=\sigma=0, \kappa_{1}=f, \kappa_{2}=g .
$$

Substituting these and the curvature $K^{\mathbb{R}^{2}}=0$ into Equation (4) we conclude that the Riemannian submersion $\pi$ is biharmonic if and only if

$$
\begin{aligned}
& \Delta^{M} f=0, \\
& \Delta^{M} g=0 .
\end{aligned}
$$

Looking for the special solutions of the form $\ln \beta=\int \varphi(x) d x+\int \phi(y) d y$ we have $f=\varphi(x)$ and $g=\phi(y)$. Substituting these into system (12) we obtain a system of ODE:

$$
\begin{aligned}
& \varphi(x) \varphi^{\prime}(x)-\varphi^{\prime \prime}(x)=0, \\
& \phi(y) \phi^{\prime}(y)-\phi^{\prime \prime}(y)=0 .
\end{aligned}
$$

which has solutions $\varphi(x)=\frac{c_{1}\left(1+e^{c_{1} x}\right)}{1-e^{c_{1} x}}$, and $\phi(y)=\frac{b_{1}\left(1+e^{b_{1} y}\right)}{1-e^{b_{1} y}}$, from which we obtain the example.

Example 2. The Riemannian submersion from Nil space

$$
\begin{gathered}
\pi:\left(\mathbb{R}^{3}, g_{N i l}=\mathrm{d} x^{2}+\mathrm{d} y^{2}+(\mathrm{d} z-x \mathrm{~d} y)^{2}\right) \rightarrow\left(\mathbb{R}^{2}, d x^{2}+\left(1+x^{2}\right)^{-2} d z^{2}\right) \\
\pi(x, y, z)=(x, z)
\end{gathered}
$$

is not a biharmonic map.

We can check that $e_{1}=\frac{\partial}{\partial x}, e_{2}=-\frac{x}{\sqrt{1+x^{2}}} \frac{\partial}{\partial y}-\sqrt{1+x^{2}} \frac{\partial}{\partial z}, e_{3}=\frac{1}{\sqrt{1+x^{2}}} \frac{\partial}{\partial y}$ form an orthonormal frame on Nil space adapted to the Riemannian submersion with $d \pi\left(e_{3}\right)=0, \quad d \pi\left(e_{i}\right)=\varepsilon_{i}$, for $i=1,2$ for an orthonormal frame $\varepsilon_{1}=\frac{\partial}{\partial x}, \varepsilon_{2}=$ $-\sqrt{1+x^{2}} \frac{\partial}{\partial z}$ on the base space. We can compute the Lie brackets as

$$
\begin{array}{r}
{\left[e_{1}, e_{2}\right]=\frac{x}{1+x^{2}} e_{2}-\frac{1-x^{2}}{1+x^{2}} e_{3}} \\
{\left[e_{1}, e_{3}\right]=-\frac{x}{1+x^{2}} e_{3}, \quad\left[e_{2}, e_{3}\right]=0,}
\end{array}
$$

from which we obtain the integrability data of the Riemannian submersion $\pi$ as $f_{1}=0, f_{2}=\frac{x}{1+x^{2}}, \quad \kappa_{1}=-\frac{x}{1+x^{2}}, \quad \sigma=\frac{1-x^{2}}{2\left(1+x^{2}\right)}, \quad \kappa_{2}=0$. Since $\kappa_{2}=0$, we apply 
Corollary 2.2 to conclude that $\pi$ is biharmonic if and only if Equation (11) holds. However, a simple computation shows that the left-hand side of the first equation of (11) equals $\frac{x^{3}-7 x}{\left(1+x^{2}\right)^{3}}$ which does not vanish identically. Thus, the Riemannian submersion $\pi$ is not biharmonic.

\section{Proofs of the MAIN RESUlts}

In this section we will give a complete classification of biharmonic Riemannian submersions from a 3-dimensional space form. This is accomplished by choosing a special adapted orthonormal frame that exists on a space form which simplifies the biharmonic equation drastically. We will use the notations $M^{3}(c)$ for a space form with constant sectional curvature $c$ and $R_{i j k l}=-\left\langle R\left(e_{i}, e_{j}\right) e_{k}, e_{l}\right\rangle$ for the components of curvature with respect to an orthonormal basis. The following lemmas will be used to prove the main theorems.

Lemma 3.1. Let $\pi: M^{3}(c) \longrightarrow\left(N^{2}, h\right)$ be a Riemannian submersion from a space form of constant sectional curvature $c$. Then, there exists an orthonormal frame $\left\{e_{1}, e_{2}, e_{3}\right\}$ on $M^{3}(c)$ adapted to the Riemannian submersion such that all the integrability data $f_{1}, f_{2}, \kappa_{1}, \kappa_{2}$ and $\sigma$ are constant along fibers of $\pi$, i.e.,

$$
e_{3}\left(f_{1}\right)=e_{3}\left(f_{2}\right)=e_{3}\left(\kappa_{2}\right)=e_{3}\left(\kappa_{1}\right)=e_{3}(\sigma)=0 .
$$

Proof. By definition, $f_{i}=F_{i} \circ \pi$ for $i=1,2$, so they are constant along the fibers. It remains to show that

$$
e_{3}\left(\kappa_{2}\right)=0, \quad e_{3}\left(\kappa_{1}\right)=0, \quad e_{3}(\sigma)=0 .
$$

One can easily check that the Jacobi identity applies to the frame $\left\{e_{1}, e_{2}, e_{3}\right\}$ yields

$$
2 e_{3}(\sigma)+\kappa_{1} f_{1}+\kappa_{2} f_{2}+e_{2}\left(\kappa_{1}\right)-e_{1}\left(\kappa_{2}\right)=0 .
$$

A straightforward computation using (15) and the fact that $M^{3}(c)$ has constant sectional curvature $c$ gives

$$
\left\{\begin{array}{l}
R_{1312}^{M}=-\left(e_{1}(\sigma)-2 \kappa_{1} \sigma\right)=0, \\
R_{1313}^{M}=-\left[-e_{1}\left(\kappa_{1}\right)-\sigma^{2}+\kappa_{1}^{2}-\kappa_{2} f_{1}\right]=c \\
R_{1323}^{M}=-\left[-e_{1}\left(\kappa_{2}\right)+e_{3}(\sigma)+\kappa_{1} f_{1}+\kappa_{1} \kappa_{2}\right]=0 \\
R_{1212}^{M}=-\left(e_{2}\left(f_{1}\right)-e_{1}\left(f_{2}\right)+f_{1}^{2}+f_{2}^{2}+3 \sigma^{2}\right)=c \\
R_{1223}^{M}=-\left(e_{2}(\sigma)-2 \kappa_{1} \kappa_{2}\right)=0 \\
R_{2313}^{M}=-\left(-e_{2}\left(\kappa_{1}\right)-e_{3}(\sigma)-\kappa_{2} f_{2}+\kappa_{1} \kappa_{2}\right)=0 \\
R_{2323}^{M}=-\left(-\sigma^{2}-e_{2}\left(\kappa_{2}\right)+\kappa_{1} f_{2}+\kappa_{2}^{2}\right)=c .
\end{array}\right.
$$


Applying $e_{3}$ to both sides of the fourth equation of (16) and using (15), together with $e_{3} e_{1}=\left[e_{3}, e_{1}\right]+e_{1} e_{3}$ and $e_{3} e_{2}=\left[e_{3}, e_{2}\right]+e_{2} e_{3}$, we get

$$
\sigma e_{3}(\sigma)=0
$$

which implies

$$
e_{3}(\sigma)=0
$$

Using this and applying $e_{3}$ to both sides of the 1 st and the 5 th equation of (16) separately, we obtain

$$
e_{3}\left(\kappa_{1}\right)=0, e_{3}\left(\kappa_{2}\right)=0
$$

which completes the proof of the lemma.

Lemma 3.2. Let $\pi:\left(M^{3}(c), g\right) \longrightarrow\left(N^{2}, h\right)$ be a Riemannian submersion with an adapted frame $\left\{e_{1}, e_{2}, e_{3}\right\}$ and the integrability data $f_{1}, f_{2}, \kappa_{1}, \kappa_{2}$ and $\sigma$. Then, there exists another adapted orthonormal frame $\left\{e_{1}^{\prime}, e_{2}^{\prime}, e_{3}^{\prime}=e_{3}\right\}$ on $M^{3}(c)$ with integrability data $f_{1}^{\prime}, f_{2}^{\prime}, \kappa_{1}^{\prime}=\sqrt{\kappa_{1}^{2}+\kappa_{2}^{2}}, \kappa_{2}^{\prime}=0$, and $\sigma^{\prime}=\sigma$.

Proof. Choose an orthonormal frame $\left\{e_{1}, e_{2}, e_{3}\right\}$ on $M^{3}(c)$ adapted to the Riemannian submersion $\pi$. It follows from Lemma 3.1 that the integrability data $\kappa_{1}$ and $\kappa_{2}$ are constant along the fibers of $\pi$. By a well-known fact from topology that there exist functions $\overline{\kappa_{1}}$ and $\overline{\kappa_{2}} \in C^{\infty}(N)$ such that $\kappa_{1}=\overline{\kappa_{1}} \circ \pi$ and $\kappa_{2}=$ $\overline{\kappa_{2}} \circ \pi$. Suppose $e_{1}, e_{2}$ are $\pi$-related to $\varepsilon_{1}, \varepsilon_{2}$ respectively. Then, it is easy to

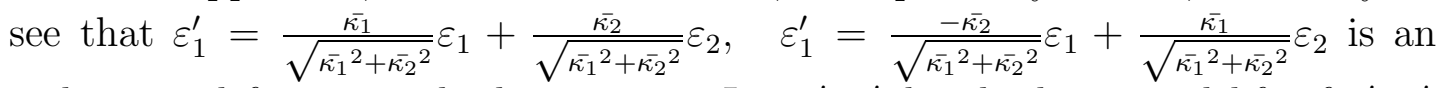
orthonormal frame on the base space. Let $e_{1}^{\prime}, e_{2}^{\prime}$ be the horizontal lift of $\varepsilon_{1}^{\prime}, \varepsilon_{2}^{\prime}$ respectively. Then, one can easily check that the adapted orthonormal frame $\left\{e_{1}^{\prime}, e_{2}^{\prime}, e_{3}\right\}$ satisfies the required conditions stated in the lemma.

Now we are ready to give the following classification of biharmonic Riemannian submersions.

Theorem 3.3. Let $\pi:\left(M^{3}(c), g\right) \longrightarrow\left(N^{2}, h\right)$ be Riemannian submersion from a space form of constant sectional curvature $c$. Then, $\pi$ is biharmonic if and only if it is harmonic.

Proof. By Lemma 3.2, we can choose an orthonormal frame $\left\{e_{1}, e_{2}, e_{3}\right\}$ adapted to the Riemannian submersion with integrability data $\left\{f_{1}, f_{2}, \kappa_{1}, \kappa_{2}, \sigma\right\}$ with 
$\kappa_{2}=0$. With respect to this frame the curvature equation (16) reduces to

$$
\left\{\begin{array}{l}
e_{1}(\sigma)-2 \kappa_{1} \sigma=0 \\
-\left[-e_{1}\left(\kappa_{1}\right)-\sigma^{2}+\kappa_{1}^{2}\right]=c \\
\kappa_{1} f_{1}=0 \\
-\left(e_{2}\left(f_{1}\right)-e_{1}\left(f_{2}\right)+f_{1}^{2}+f_{2}^{2}+3 \sigma^{2}\right)=c \\
e_{2}(\sigma)=0 \\
e_{2}\left(\kappa_{1}\right)=0 \\
-\left(-\sigma^{2}+\kappa_{1} f_{2}\right)=c
\end{array}\right.
$$

By the 3rd equation in (17), we have either $\kappa_{1}=0$ or $f_{1}=0$. For the first case, $\kappa_{1}=0$, then, by (6), the tension fields of $\pi$ vanishes and the hence the Riemannian submersion is harmonic. For the second case, $\kappa_{1} \neq 0$, then we have $f_{1}=0$. We will show that this latter case cannot happen. We will prove this by using proof by contradiction in the following two cases:

Case I: $\kappa_{1} \neq 0, \quad f_{1}=0$ and $f_{2}=0$. In this case, the 4 th and the 7 th equations in (17) implies that $\sigma=c=0$. Now substituting $f_{1}=f_{2}=\sigma=0$ and $\kappa_{2}=0$ into biharmonic equation (11) we obtain

$$
\Delta \kappa_{1}=0,
$$

which, by a straightforward computation using (5), the 2nd, and the 6th equations of (17), can be turned into

$$
\kappa_{1}^{3}=0
$$

It follows that $\kappa_{1}=0$ which is a contradiction.

Case II: $\kappa_{1} \neq 0, f_{1}=0$ and $f_{2} \neq 0$. In this case, we use $f_{1}=0$ and the 5 th, the 6 th and the 7 th equations of (17) to reduce the biharmonic equation (11) into

$$
-\Delta^{M} \kappa_{1}+\kappa_{1}\left\{-c-3 \sigma^{2}+f_{2}^{2}\right\}=0,
$$

where we have used the fact that the Gauss curvature of the target surface $K^{N}=$ $c+3 \sigma^{2}$ obtained from $\mathrm{O}^{\prime}$ Neill's curvature formula for a Riemannian submersion ([13]). A straightforward computation gives

$$
\begin{aligned}
-\Delta^{M} \kappa_{1} & =-e_{1} e_{1}\left(\kappa_{1}\right)+\nabla_{e_{2}} e_{2}\left(\kappa_{1}\right)+\nabla_{e_{3}} e_{3}\left(\kappa_{1}\right) \\
& =-e_{1}\left(\kappa_{1}^{2}-\sigma^{2}+c\right)+f_{2} e_{1}\left(\kappa_{1}\right)+\kappa_{1} e_{1}\left(\kappa_{1}\right) \\
& =5 \kappa_{1} \sigma^{2}-\kappa_{1}^{3}-\kappa_{1} c+f_{2}\left(\kappa_{1}^{2}-\sigma^{2}+c\right) .
\end{aligned}
$$


Substituting this into (18) and simplifying the resulting equation we get

$$
\kappa_{1}\left(3 \sigma^{2}-\kappa_{1}^{2}-3 c\right)=0 .
$$

By assumption, $\kappa_{1} \neq 0$, so (19) implies that

$$
\kappa_{1}^{2}=3 \sigma^{2}-3 c .
$$

Applying $e_{1}$ to both sides of (20) yields

$$
\kappa_{1} e_{1}\left(\kappa_{1}\right)=3 \sigma e_{1}(\sigma) .
$$

Combining this and the 1st and the 2nd equations in (17) we obtain

$$
\kappa_{1}\left(\kappa_{1}^{2}-\sigma^{2}+c\right)=6 \kappa_{1} \sigma^{2},
$$

which, since $\kappa_{1} \neq 0$, is equivalent to

$$
\left(\kappa_{1}^{2}-\sigma^{2}+c\right)=6 \sigma^{2},
$$

or

$$
\kappa_{1}^{2}=7 \sigma^{2}-c .
$$

Similarly, applying $e_{1}$ to both sides of (21) and using the 1 st and the 2nd equations in (17) we get

$$
\kappa_{1}^{2}=15 \sigma^{2}-c
$$

Combining (20), (21) with (22) we have $\kappa_{1}=\sigma=c=0$. In particular, $\kappa_{1}=0$, which contradicts our assumption. Thus, we complete the proof of the theorem.

Corollary 3.4. (1) If $\pi: \mathbb{R}^{3} \longrightarrow\left(N^{2}, h\right)$ is a biharmonic horizontally homothetic submersion from Euclidean space, then $\left(N^{2}, h\right)$ is flat and $\pi$ is a composition of an orthogonal projection $\mathbb{R}^{3} \longrightarrow \mathbb{R}^{2}$ followed by a covering map $\mathbb{R}^{2} \longrightarrow\left(N^{2}, h\right)$;

(2) There exists no biharmonic Riemannian submersion $\pi: \mathbb{H}^{3} \longrightarrow\left(N^{2}, h\right)$ no matter what $\left(N^{2}, h\right)$ is.

Proof. By a theorem in [14], a horizontally homothetic submersion $\pi: \mathbb{R}^{3} \longrightarrow$ $\left(N^{2}, h\right)$ is a Riemannian submersion up to a homothety. It follows from our Theorem 3.3 that $\pi$ has to be harmonic and hence a harmonic morphism (see, e.g., [1]). Using Baird-Wood's Bernstein theorem [2] for harmonic morphisms we conclude that $\pi$ is a composition of an orthogonal projection $\mathbb{R}^{3} \longrightarrow \mathbb{R}^{2}$ followed by a weakly conformal map $\mathbb{R}^{2} \longrightarrow\left(N^{2}, h\right)$. Since $\pi$ is a Riemannian submersion, the dilation of the composition has to be constant 1 , from which we conclude that the weakly conformal map has to be a covering map and $\left(N^{2}, h\right)$ has to be flat. This gives the Statement (1). For Statement (2), we first note that the biharmonic Riemannian submersion $\pi: \mathbb{H}^{3} \longrightarrow\left(N^{2}, h\right)$ is a harmonic morphism 
because, by Theorem 3.3, it is harmonic. Using again Baird-Wood's Bernstein theorem [3] we conclude that $\pi$ is the composition of a the orthogonal projection $\mathbb{H}^{3} \longrightarrow \mathbb{H}^{2}$, or the projection to the plane at infinity $\mathbb{H}^{3} \longrightarrow \mathbb{C}$, followed by a weakly conformal map $\rho: \mathbb{H}^{2} \longrightarrow\left(N^{2}, h\right)$, or $\rho: \mathbb{C} \longrightarrow\left(N^{2}, h\right)$, respectively. Suppose the projection has dilation $\lambda_{1}$ and the conformal factor of the weakly conformal map is $\lambda_{2}$, then, the composition map has dilation $\lambda_{1}\left(\lambda_{2} \circ \pi\right)$. It follows from [8] that in both cases, $\lambda_{1}$ is not constant along the fibers, however, it is clear that $\left(\lambda_{2} \circ \pi\right)$ is constant along the fibers. It follows that the product $\lambda_{1}\left(\lambda_{2} \circ \pi\right)$ cannot be 1 , i.e, in either case, the map cannot be a Riemannian submersion. This completes the proof of the corollary.

\section{REFERENCES}

[1] P. Baird and J. C. Wood, Harmonic morphisms between Riemannian manifolds, London Math. Soc. Monogr. (N.S.) No. 29, Oxford Univ. Press (2003).

[2] P. Baird and J. C. Wood, Bernstein theorems for harmonic morphisms from $R^{3}$ and $S^{3}$, Math. Ann. 280 (1988), no. 4, 579-603.

[3] P. Baird and J. C. Wood, Harmonic morphisms and conformal foliations by geodesics of three-dimensional space forms, J. Austral. Math. Soc. Ser. A 51 (1991), no. 1, 118-153.

[4] R. Caddeo, S. Montaldo and C. Oniciuc, Biharmonic submanifolds in spheres, Israel J. Math. 130 (2002), 109-123.

[5] B. Y. Chen, Some open problems and conjectures on submanifolds of finite type, Soochow J. Math. 17 (1991), no. 2, 169-188.

[6] B. Y. Chen and S. Ishikawa, Biharmonic pseudo-Riemannian submanifolds in pseudoEuclidean spaces, Kyushu J. Math. 52 (1998), no. 1, 167-185.

[7] I. Dimitrić, Submanifolds of $E^{m}$ with harmonic mean curvature vector, Bull. Inst. Math. Acad. Sinica 20 (1992), no. 1, 53-65.

[8] S. Gudmundsson, The geometry of harmonic morphisms, Ph. D thesis, University of Leeds, 1992.

[9] T. Hasanis and T. Vlachos, Hypersurfaces in $E^{4}$ with harmonic mean curvature vector field, Math. Nachr. 172 (1995), 145-169.

[10] G. Y. Jiang, 2-Harmonic maps and their first and second variational formulas, Chin. Ann. Math. Ser. A 7(1986) 389-402.

[11] G. Y. Jiang, Some non-existence theorems of 2-harmonic isometric immersions into Euclidean spaces, Chin. Ann. Math. Ser. 8A (1987) 376-383.

[12] E. Loubeau and Y. -L. Ou, Biharmonic maps and morphisms from conformal mappings, Tôhoku Math J., to appear, 2010.

[13] B. O'Neill, The fundamental equations of a submersion, Michigan Math. J. 131966 459469.

[14] Y. -L. Ou and G. Walschap, A classification of horizontally homothetic submersions from space forms of nonnegative curvature, Bull. of London Math. Soc., 38(3) (2006), 485-493. 
Department of Mathematics \& Physics,

YunNan Wenshan University,

No. 2 Xuefu Road Wenshan County Wenshan, Yunnan 653000,

PeOple's Republic of China

E-MAIL:ZEPING.WANG@GMAIL.COM (WANG),

Department of MATHEMATiCs,

Texas A \& M University-Commerce,

COMMERCE TX 75429,

USA.

E-MAIL:YELIN_OU@TAMU-COMMERCE.EDU (OU) 